\title{
A NOTE ON LINEAR VECTOR SPACES OF MAPPINGS WITH POSITIVE JACOBIANS
}

\section{MICHAEL GOLOMB}

Let $\mathfrak{F}$ be a family of continuously differentiable mappings $f$ of an open set $D$ in the $x y$-plane into the $u v$-plane satisfying the following conditions:

(a) $f, g \in \mathfrak{F} \rightarrow \lambda f+\mu g \in \mathfrak{F}$ for all real $\lambda, \mu$,

(b) $f \in \mathfrak{F} \rightarrow$ the Jacobian of $f$ is non-negative and zero only if its rank is zero,

(c) $\mathfrak{F}$ contains two mappings $f_{1}=\left(u_{1}, v_{1}\right), f_{2}=\left(u_{2}, v_{2}\right)$ such that the Jacobian

for $(x, y) \in D$.

$$
\frac{\partial\left(v_{1}, v_{2}\right)}{\partial(x, y)} \neq 0
$$

The mappings described by functions $f=u+i v$ regular analytic in $z=x+i y,(x, y) \in D$, form such a family. J. E. McLaughlin and C. J. Titus [1] proved the converse: If the two mappings of condition (c) are described by functions regular analytic in $z=x+i y$, then $\mathfrak{F}$ is a linear vector space of functions regular analytic in $z=x+i y$, $(x, y) \in D$. It is the purpose of this note to give a characterization of the general class $\mathfrak{F}$.

Consider continuously differentiable solutions $u(x, y), v(x, y)$ of the system of partial differential equations

$$
L_{1} \equiv u_{x}-a_{11} v_{x}-a_{12} v_{y}=0, \quad L_{2} \equiv u_{y}+a_{21} v_{x}+a_{22} v_{y}=0
$$

where $a_{11}, a_{12}, a_{21}, a_{22}$ are real functions of $x, y$ continuous in $D$. If the system is elliptic [2], $\left(a_{11}+a_{22}\right)^{2}<4 a_{12} a_{21}$ and $a_{12}>0, a_{21}>0$ in $D$, then the Jacobian

$$
\frac{\partial(u, v)}{\partial(x, y)}=a_{21} v_{x}^{2}+\left(a_{11}+a_{22}\right) v_{x} v_{y}+a_{12} v_{y}^{2}
$$

is positive unless it is of rank 0 . Therefore, the mappings $f$ described by functions $u, v$ continuously differentiable in $D$ which are solutions of the elliptic system (1) form a family $\mathfrak{F}$. We prove the converse.

THEOREM. The mappings $f$ of a family $\mathfrak{F}$ are described by functions $u, v$ that are solutions of a unique elliptic system of form (1).

Received by the editors December 31, 1953. 
To prove this consider the mappings $f_{1}, f_{2}$ satisfying condition (c). Then we can solve the system

$$
u_{i x}-a_{11} v_{i x}-a_{12} v_{i y}=0, \quad u_{i y}+a_{21} v_{i x}+a_{22} v_{i y}=0 \quad(i=1,2)
$$

for $a_{11}, a_{12}, a_{21}, a_{22}$, and the solution is uniquely determined. The system

$$
L_{1} \equiv u_{x}-a_{11} v_{x}-a_{12} v_{y}=0, \quad L_{2} \equiv u_{y}+a_{21} v_{x}+a_{22} v_{y}=0
$$

is then satisfied by the linear combinations $(u, v)$ of $\left(u_{1}, v_{1}\right),\left(u_{2}, v_{2}\right)$ with real constant coefficients, and since the corresponding mappings $f$ are in $\mathfrak{F}$ we have by (b)

$$
\frac{\partial(u, v)}{\partial(x, y)}=a_{21} v_{x}^{2}+\left(a_{11}+a_{22}\right) v_{x} v_{y}+a_{12} v_{y}^{2}>0
$$

except where $v_{x}=v_{y}=0$. Since every real vector $(X, Y)$ is a linear combination with real coefficients of the linearly independent vectors $\left(v_{1 x}, v_{1 y}\right),\left(v_{2 x}, v_{2 y}\right)$, the form $a_{21} X^{2}+\left(a_{11}+a_{22}\right) X Y+a_{12} Y^{2}$ is positive definite, $\left(a_{11}+a_{22}\right)^{2}<4 a_{12} a_{21}$, and $a_{12}>0, a_{21}>0$.

Now let $f=(u, v)$ be any mapping in class $\mathfrak{F}$. By Lemma 2 of [1] we know that

$$
J(f)=\lambda_{1} J\left(f_{1}\right)+\lambda_{2} J\left(f_{2}\right)
$$

where $J(f), J\left(f_{1}\right), J\left(f_{2}\right)$ are the Jacobian matrices of $f, f_{1}, f_{2}$ and $\lambda_{1}, \lambda_{2}$ are scalars that may depend on $x, y$. It follows that $u, v$ satisfy the system

$$
L_{1}=0, \quad L_{2}=0,
$$

and the theorem is proved.

REMARK 1. If the two mappings of condition (c) are described by functions regular analytic in $z=x+i y$, then $L_{1}=0, L_{2}=0$ are the Cauchy-Riemann equations and we obtain the characterization of the analytic functions given in [1].

REMARK 2. If the two mappings of condition (c) are described by sigma-monogenic functions [3] $f=u+i v$, where

$$
\sigma_{1}(x) u_{x}=\tau_{1}(y) v_{y}, \quad \sigma_{2}(x) u_{y}=-\tau_{2}(y) v_{x}
$$

with $\sigma_{i}>0, \tau_{i}>0$, then $\mathfrak{F}$ contains only sigma-monogenic functions.

REMARK 3. If the two mappings of condition (c) are described by functions $u_{i}, v_{i}$ analytic in the real variables $x, y$, then the coefficients $a_{i j}$ of the system $L_{1}=0, L_{2}=0$ are analytic, and by a well known property of such systems, $\mathfrak{F}$ contains only mappings described by functions analytic in $x, y$. 


\section{REFERENCES}

1. J. E. McLaughlin, and C. J. Titus, A characterization of analytic functions, Proc. Amer. Math. Soc. vol. 5 (1954) pp. 348-351.

2. R. Courant and D. Hilbert, Methoden der mathematischen Physik, vol. II, Berlin, 1937; see p. 143.

3. Lipman Bers, The expansion theorem for sigma-monogenic functions, Amer. J. Math. vol. 72 (1950) pp. 705-712.

Purdue University 